\title{
Krypton K-Shell X-Ray Spectra Recorded by the HENEX Spectrometer
}

\author{
J. F. Seely ${ }^{1 *}$, C. A. Back ${ }^{2}$, C. Constantin ${ }^{2}$, R. W. Lee ${ }^{2}$, H.-K. Chung, L. T. Hudson ${ }^{3}$, \\ C. I. Szabo ${ }^{3}$, A. Henins ${ }^{3}$, G. E. Holland ${ }^{4}$, R. Atkin ${ }^{5}$, and L. Marlin'
}

\author{
${ }^{1}$ Naval Research Laboratory, Space Science Division, Washington DC 20375 \\ ${ }^{2}$ Lawrence Livermore National Laboratory, Livermore CA 94550 \\ ${ }^{3}$ National Institute of Standards and Technology, Gaithersburg MD 20899 \\ ${ }^{4}$ SFA Inc., 9315 Largo Drive West Suite 200, Largo MD 20774 \\ 'Tiger Innovations, L.L.C., 3610 Vacation Lane, Arlington VA. 22207
}

4 January 2005

\begin{abstract}
High resolution $\mathrm{x}$-ray spectra were recorded by the High Energy Electronic X-Ray (HENEX) spectrometer from a variety of targets irradiated by the Omega laser at the Laboratory for Laser Energetics. The HENEX spectrometer utilizes four reflection crystals covering the $1 \mathrm{keV}$ to $20 \mathrm{keV}$ energy range and one quartz(10-11) transmission crystal (Laué geometry) covering the $11 \mathrm{keV}$ to $40 \mathrm{keV}$ range. The time-integrated spectral images were recorded on five CMOS $x$-ray detectors. In the spectra recorded from krypton-filled gasbag and hohlraum targets, the helium-like K-shell transitions n=1$2,1-3$, and $1-4$ appeared in the $13 \mathrm{keV}$ to $17 \mathrm{keV}$ energy range. A number of additional spectral features were observed at energies lower than the helium-like $n=1-3$ and $n=1-4$
\end{abstract}


transitions. Based on computational simulations of the spectra using the FLYCHK/FLYSPEC codes, which included opacity effects, these additional features are identified to be inner-shell transitions from the Li-like through N-like krypton charge states. The comparisons of the calculated and observed spectra indicate that these transitions are characteristic of the plasma conditions immediately after the laser pulse when the krypton density is $2 \times 10^{18} \mathrm{~cm}^{-3}$ and the electron temperature is in the range 2.8 $\mathrm{keV}$ to $3.2 \mathrm{keV}$. These spectral features represent a new diagnostic for the charge state distribution, the density and electron temperature, and the plasma opacity. Laboratory experiments indicate that it is feasible to record $\mathrm{K}$-shell spectra from gold and higher $\mathbf{Z}$ targets in the $>60 \mathrm{keV}$ energy range using a Ge(220) transmission crystal.

Key Words: x-ray spectra, K-shell transitions, krypton, Laué geometry, crystal spectrometer

"Corresponding author, john.secly anrl navy mil, Tel. 202-767-3529. 


\section{Introduction}

The use of a transmission crystal (Laue diffraction) has proven to be a promising new technique for recording hard $x$-ray spectra from laser-produced plasmas. The initial spectrometer, called the Hard X-Ray Spectrometer (HXS), utilized a quartz(10-11) erystal bent to a $112 \mathrm{~mm}$ radius of curvature and covering the $12 \mathrm{keV}$ to $60 \mathrm{keV}$ energy range [1]. This spectrometer was used to record time-integrated $\mathrm{x}$-ray spectra from a number of targets irradiated by the Omega laser at the Laboratory for Laser Energetics. The spectral resolving power was $\approx 100$, limited by the CCD detector resolution and spreading in its phosphor coating. K-shell spectral features were observed from elements up to silver $(Z=47)$ near $22 \mathrm{kcV}$ and $\mathrm{L}$-shell features from elements up to uranium $(Z=92)$ in the $12 \mathrm{keV}$ to $22 \mathrm{keV}$ range [2]. The analysis of the spectra indicated that the uranium spectra were inner-shell transitions in the Se-like and nearby charge states, probably generated by energetic electrons, and that opacify effects were important.

Based on those successful experiments, a new spectrometer was designed and built that is named HENEX (High Energy Electronic X-Ray Spectrometer). HENEX implements four reflection erystals covering the $1 \mathrm{keV}$ to $20 \mathrm{keV}$ range and one quartu(10-11) transmission (Laué) erystal covering $11 \mathrm{keV}$ to $40 \mathrm{keV}$. The transmission crystal is bent to a $119 \mathrm{~mm}$ radius of eurvature and provides $\geq 300$ resolving power when recording the spectra on a phosphor-coated CMOS detector.

The improved resolution of the HENEX transmission erystal spectra has permitted for the first time the observation and identification of finc details in the krypton $K$-shell spectra in the $13 \mathrm{keV}$ to $17 \mathrm{keV}$ energy range. The targets were gasbags or hohlraums filled with $0.5 \mathrm{~atm}$ to $0.8 \mathrm{~atm}$ of $100 \% \mathrm{Kr}$. In addition to the He-like 
resonance transitions ( $\mathrm{n}=1-2,1-3$, and $1-4$ ), inner-shell transitions from the $\mathrm{Li}$-like through $\mathrm{N}$-like krypton charge states, appearing in the spectra on the low-energy sides of the He-like $n=1-3$ and $n=1-4$ transitions, have been identified. Simulations using the FLYCHK/FL YSPEC codes indicate that the relative intensities of the He-like through Nlike transitions are consistent with the plasma conditions immediately after the laser pulse when the krypton density is $2 \times 10^{18} \mathrm{~cm}^{-3}$, a factor of 10 lower than the initial gas density, and the electron temperature is in the range $2.8 \mathrm{keV}$ to $3.2 \mathrm{keV}$. These spectral features represent a new plasma diagnostic for the charge state distribution, density, electron temperature, and opacity.

In experiments using a laboratory $x$-ray source with a tungsten anode, the characteristic W x-ray lines were observed in the $59 \mathrm{keV}$ to $67 \mathrm{keV}$ range using a $\mathrm{Ge}(220)$ transmission crystal. The $K \alpha_{1}$ and $K \alpha_{2}$ lines and the $K \beta_{1}$ and $K \beta_{2}$ lines were well resolved. This laboratory work indicates that it is feasible to design and field a transmission crystal spectrometer to record $\mathrm{K}$-shell spectra from laser-irradiated gold and higher $Z$ targets. K-shell emission from gold targets was recently obscrved at the National Ignition Facility (NIF) using filtered $\mathrm{x}$-ray diodes [3]. Recording the K-shell spectra of gold and heavier elements using a transmission erystal spectrometer would permit the implementation of K-shell plasma diagnostic techniques in NIF temperature, density, and opacity regimes not presently accessible by existing instrumentation [4]:

\section{Spectrometer Design and Laboratory Calibrations}

The HENEX spectrometer, though commissioned as a core-level diagnostic for NIF, was designed to fit onto a TIM instrument module at LLE and is shown schematically in Fig. 1. The instrument consists of a spectrometer box housing the five 
crystals and five CMOS detectors, rechargeable lithium ion battery pack that provides power to the detectors and on-board electronies, and drive electronics that automatically reads and stores the five spectral images immediately after the laser shot. Not shown in Fig. 1 is the nosecone that has shielded lines of sight to the five erystals that are at a standoff distance of $0.5 \mathrm{~m}$ from the Omega target.

The communications between the laboratory-based control computer and the instrument in the TIM are by fiber optics. Two fiber optic cables are used to up-load commands, including the Omega facility aming and shot fiducials, and to down-load the spectral and dark (unilluminated) images immediately after the shot. The HENEX spectrometer is sapable of autonomous operations limited only by weekly battery charges.

The HENEX instrument was constructed with electromagnetic interference (EMI) shielding sa that it would be unhamed during the laser shots. This was accomplished by enclosing the entire instrument in an electrically conductive skin which serves as a Faraday cage. Since the instrument is intemally powered by the battery pack and communications are via fiber optics, there is no electrically conductive connection to the instrument in the TIM. No EMI was observed during the week of Onega laser shots. It is expected that EMI would not occur at larger laser facilities such as NIF.

A detailed description of the HENEX spectrometer design, capabilities, and laboratory calibrations of all five crystal channels will be published elsewhere [5]. Shown in Fig. 2 is a comparison of a gold spectrum recorded by the transmission crystal using a Laboratory $x$-ray source with a gold anode and the spectrum recorded from a gold target irradiated by the Omega laser. The target was a gold half hohtraum, and 23 Omega beams 
with $10.8 \mathrm{~kJ}$ total energy were incident through the open end of the hohlraum [3]. The $L \beta_{1}, L \beta_{2}$, and $L \gamma_{12}$ characteristic $x$-ray lines, from neutral gold atoms, are identified in the laboratory spectrum. The spectrum from the gold hohltaum has a broad and intense feature centered near $12 \mathrm{keV}$, at higher energy than the $L \beta_{1}, L \beta_{2}$ lines, and multiple weak but well-resolved fcatures near $\mathrm{L} \gamma_{1,2}$. The features in the gold hohlraum spectrum are inner-shell $\mathrm{L}$-shell transitions in highly charged $\mathrm{A}\lrcorner$ fons and are analogous to the $\mathrm{U}$ and $\mathrm{Pb} \mathrm{L}$-shell features previously observed and identified [2].

\section{Krypton K-Shell Spectra}

A number of krypton spectra were recorded from Kr-filled gasbag and hohIraum targets irradiated by the Omega laser. Two representative spectral inages are shown in Fig. 3. The gasbag target was filled with $0.5 \mathrm{~atm}$ to $0.8 \mathrm{~atm}$ of $100 \% \mathrm{KF}$ and was irradiated by 49 Omega beams with $21.6 \mathrm{~kJ}$ total energy. The hohlraum was filled with $0.7 \mathrm{~atm}$ of $100 \% \mathrm{Kr}$ and was irradiated by 40 Omega beams with $18.9 \mathrm{~kJ}$.

The spectral images shown in Fig. 3 were acquired with the HENEX transmission channel. The $x$-ray energy is approximately $11 \mathrm{keV}$ at the loft and right edges of the image and increases toward the center. The image is a representation of two mirror spectra due to the symmetry of the optical design about the vertical center line. A pinhole image of the target, produced by a pinhole aperture on the central axis of the instrument, appears on the vertical center line of the top image in Fig. 3 and is used to insure sourceinstrument alignment and reproducibility.

The krypton $\mathrm{K}$-shell features are identified in the spectral images shown in Fig. 3. Three types of filters ( $\mathrm{Se}, \mathrm{Zr}$, and $\mathrm{Cd}$ ) were located between the crystal and detector, covering portions of the images in the vertical direction. Attenuation by the filters 
resulted in good exposure of the intense $\mathrm{Kr} n=1-2$ resonance line. In the vertical regions of the spectral images that had no filtration, the $K r n=1-2$ line is saturated and the weaker $\mathrm{Kr}$ lines have good exposure. Two atomic-absorption edges are identified in Fig. 3 due to the $\mathrm{K}$ edges of a Se filter at $12.65 \mathrm{keV}$ and a $\mathrm{Zr}$ filter at $18.00 \mathrm{keV}$. In other spectral Inages from higher $Z$ targets with brighter $x$-ray continua, the $\mathrm{Cd} 26.71 \mathrm{keV}$ absorption edge was observed. The filter edges, as well as the spectrometer geometry and the laboratory spectra of the characteristic $x$-ray lines, were used to establish the $x$-ray energy scales in the spectral images.

The spectrum from the Kr-filled gasbag, acquired by summing pixel values in the vertical direction, is shown in Fig. 4. The energy positions of the krypton characteristic (neutral atom) $\mathrm{Ka}$ line, the hydrogen-like $\mathrm{Ha}(\mathrm{n}=1-2)$ resonance line, and the helium-like

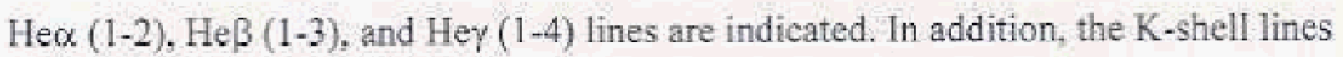
from a $\mathrm{Zn}$ backlighter are indicated between $11 \mathrm{keV}$ and $12 \mathrm{keV}$. The energy scale shown in Fig. 4 is not applicable to the $\mathrm{Zn}$ lines because the backlighter was off the spectrometer axis. However, the spectral resolution of the $\mathrm{Zn}$ lines, as well as the laboratory gold spectral lines shown in Fig. 2, are indicative of the resolving power of the instrument.

The numerous spectral features on the low-energy sides of the krypton $\mathrm{He \beta}$ and Her lines in Fig. 4 were observed for the first time using the HENEX spectrometer. These speetral features do not appear in previous krypton $\mathrm{K}$-shell spectra from $\mathrm{Kr}$-filled $\mathrm{CH}$ shell targets that were irradiated by the Omega laser [6]. They were observed but not resolved in our HXS Kr spectra [1]. These spectral features appear and are well-resolved in both the Kr-filled gasbag and hohlraum HENEX spectral images shown in Fig, 3. 
Based on the absence of the Ho resonance line in the HENEX krypton spectra, and the weak He $\beta$ and Hey lines, the spectral features on the low-energy sides of the Heß and Her lines were initially believed to be inner-shell K-shell transitions in krypton charges states lower than He-like. This identification was confirmed by FLYCHK/FLYSPEC simulations of the $\mathrm{Kr}$ spectrum.

\section{Simulated Krypton K-Shell Spectra}

As a first step in the simulation of the $\mathrm{Kr}$ spectra, the ion charge distribution was calculated using the FLYCHK code [7]. Initial estimates of the Kr density and the electron temperature were derived from the $\mathrm{Kr}$ gas fill pressure and Thomson scattering measurements. The fill pressures of $0.5 \mathrm{~atm}$ to $0.8 \mathrm{~atm}$ corresponded to $\mathrm{Kr}$ densities of $1.3 \times 10^{19} \mathrm{~cm}^{-3}$ to $2.2 \times 10^{19} \mathrm{~cm}^{-3}$. Thomson scattering data, giving values of the product of the average charge and the electron temperature $\left(Z_{2} T_{e}\right)$, indicated an electron temperature during the $1 \mathrm{~ns}$ laser pulse of $3.0 \mathrm{keV}$ when assuming $Z_{\mathrm{av}}=34$ (helium-like $\mathrm{Kr})$. At a time $0.5 \mathrm{~ns}$ after the laser pulse, the temperature was $2.3 \mathrm{keV}$ assuming $Z_{\mathrm{ar}}=26$.

The average charge was calculated for a $\mathrm{Kr}$ density of $1.76 \times 10^{19} \mathrm{~cm}^{-3}$, a range of electron temperatures from $2.0 \mathrm{keV}$ to $3.8 \mathrm{keV}$, and an optically thin plasma. The average charge was also calculated accounting for opacity over a path length of $1.2 \mathrm{~mm}$, representative of the half-width of the ellipsoidal gasbag that typically had $2.4 \mathrm{~mm}$ length and $2.75 \mathrm{~mm}$ diameter. The calculated average charge is shown in Table 1 . It is seen that in the case of the optically thick $(1.2 \mathrm{~mm})$ plasma, the average charge is approximately 32.0 at $3.0 \mathrm{keV}$ and 30.5 at $2.3 \mathrm{keV}$, differing from the corresponding values 34 and 26 initially assumed for the Thomson scattering measurements. 
Shown in Fig. 5(a) are Kr K-shell spectra summed over unfiltered (saturated Hea line) and filtered (unsaturated Heo line) regions of the HENEX spectral image. The spectra calculated by the FLYCHKJFLYSPEC codes for $2 \times 10^{19} \mathrm{~cm}^{-2} \mathrm{Kr}$ density and for electron temperatures in the $2.6 \mathrm{keV}$ to $3.4 \mathrm{keV}$ range are shown in Fig. 5 (b). Based on the calculated spectra, the spectral features are identified in Fig. 5(a) as K-shell transitions from the He-like through $\mathrm{N}$-like charge states. The most intense features in the HENEX spectra near $15 \mathrm{keV}$ energy are from the Li-like and Be-like charge states. In the calculated spectra, Fig, $5(6)$, the Li-like and Be-like features have the highest relative intensities for temperatures in the range $2.8 \mathrm{keV}$ to $3.2 \mathrm{keV}$.

Shown in Fig. 5(c) are spectra calculated for an assumed electron temperature of $3.0 \mathrm{keV}$ and for $\mathrm{Kr}$ densities from $2 \times 10^{17} \mathrm{~cm}^{-3}$ to $2 \times 10^{20} \mathrm{~cm}^{-3}$. The best agreement with the HENEX spectra is for a $\mathrm{Kr}$ density of $2 \times 10^{18} \mathrm{~cm}^{-3}$

Shown in Fig. 6 are spectra generated by time-dependent calculation performed by assuming that the population is initially in the bare nucleus state at $t=0$ and recombines at a fixed $T_{e}=3 \mathrm{keV}$. Fig. 6(a) shows the spectra calculated for times during the $1 \mathrm{~ns}$ laser pulse and (b) the spectra calculated for times after the pulse. The H-like $\mathrm{K}_{\mathrm{r}}$ lines are intense in the spectra calculated during the pulse while they are absent in the spectra after the pulse and are absent in the HENEX spectra. The spectra calculated for times of $3 \mathrm{~ns}$ to $5 \mathrm{~ns}$ are in best agreenent with the HENEX spectra.

Thus in general, the calculations indicate that the time-integrated HENEX spectra are characteristic of the recombining plasma after the laser pulse (at times $3 \mathrm{~ns}$ to $5 \mathrm{~ns}$ ), a Kr density of $2 \times 10^{13} \mathrm{~cm}^{-3}$ (a factor of 10 lower than the initial $\mathrm{Kr}$ gas density), and electron temperatures in the $2.8 \mathrm{keV}$ to $3.2 \mathrm{keV}$ range. The HENEX transmission orystal 
channel is compatible with a streak camera which would provide time-resolved spectra characteristic of the hot, dense plasma during the laser pulse as well as spectra characteristic of the lower-density plasma after the pulse.

\section{Extension to Gold and Higher Z K-Shell Spectra}

K-shell emission from gold targets was recently observed at WIF using filtered $\mathrm{x}$ ray diodes [3]. Laboratory experiments indicate that a transmission (symmetric Laué geometry) crystal can be implemented to record the $\mathrm{K}$-shell spectra from gold and higher $Z$ elements in the $>60$ keV energy range. Shown in Fig. 7 are spectra recorded using a laboratory $\mathrm{x}$-ray source with a tungsten anode and peak voltages in the $100 \mathrm{kV}$ to $160 \mathrm{kV}$ range The $\mathrm{W} \mathrm{K} \beta$ features at $67 \mathrm{keV}$ and the $\mathrm{K} \alpha$ features at $59 \mathrm{keV}$ are resolved. The crystal was Ge(220), and the source-erystal and crystal-detector distances were $1.12 \mathrm{~m}$ and $37 \mathrm{~cm}$, respectively.

Shown in Fig. 8 is the design of a spectrometer to record the gold $\mathrm{K}$-shell lines in the $68 \mathrm{keV}$ to $80 \mathrm{keV}$ range using a Ge(220) erystal. As for the HENEX transmission crystal channel, the $x$-rays are diffracted by the cylindrically bent crystal, converge through a focal slit, and diverge to the detector that is tangent to the Rowland circle. The source-cry stal and crystal-detector distances are $2.2 \mathrm{~m}$ and $61 \mathrm{~cm}$, respectively.

Recording the K-shell spectra of gold and heavier elements using a transmission crystal spectrometer would permit the implementation of K-shell plasma diagnostic techniques, of the type demonstrated here for $\mathrm{Kr}$, in the extremely high NIF temperature, density, and opacity regimes that are not presently accessible by instruments operating at lower energies. The spectrometer shown in Fig. 8 has a number of beneficial design features that are critically important for NIF deployment [4]: (1) no direct line of sight 
from the source to the detector permits $x$-ray and neutron shielding, (2) robust and proven crystal and detector technology and blast shielding, and (3) linear optical configuration that is compatible with DIM instrument modules and pointing techniques and with streak cameras. This type of hard $\mathrm{x}$-ray spectrometer will be important for diagnosing the extremely hot, dense plasmas produced by the NIF laser and for achieving and verifying the NIF milestones related to hot hohlraums and $x$-ray conversion from high Z targets.

\section{Conclusions}

Hard $\mathrm{X}$-ray spectra were recorded by the HENEX spectrometer from a variety of gasbag, hohlraum, and foil targets that were irradiated by the Omega laser. The krypton $\mathrm{K}$-shell emission in the $13 \mathrm{keV}$ to $18 \mathrm{keV}$ energy range was recorded. In addition to the $\mathrm{He}$-like lines, inner-shell $\mathrm{K}$ transitions from the Li-like to $\mathrm{N}$-like krypton charge states were observed and identified for the first time. The HENEX spectra are in good agrement with spectra simulated by the FLYCHK/FLYSPEC codes for the plasma conditions immediately following the laser pulse. This work demonstrates krypton $\mathrm{K}$ shell diagnostic techniques for charge distribution, temperature, density, and opacity. This is similar to the previous use of lower- $Z$ noble gases with lower-temperature plasmas. It is shown that it is feasible to extend these instrumentation and spectroscopic diagnostic techniques to the $K$-shell spectra of gold and higher- $Z$ elements at energies $>60 \mathrm{keV}$ that are appropriate for NIF science:

\section{Acknowledgements}

We thank the Omega laser operations and scientific staff for invaluable assistance. 


\section{References}

[1] Hudson LT, Henins A, Deslattes RD, Seely JE, Holland GE, Atkin R, Marlin L, Meyerhofer DD, and Stoeck $\downarrow C$. A high-energy $x$-ray spectrometer diagnostic for the OMEGA laser. Rev Sei Instr 2002;73:2270-2275.

[2] Seely, JF, Doron, R, Bar-Shalom, A, Hudson, LT, Stroeck1, C. Hard x-ray emission from laser-produced plasmas of $\mathrm{U}$ and $\mathrm{Pb}$ recorded by a transmission crystal spectrometer. JQSRT 2003;81:421-429.

[3] Schneider, M. Private communication, 2004.

[4] Seely J, Back C, Deslattes R, Hudson L, Holland G, Bell P, Miller M. Hard x-ray spectrometers for the National Ignition Facility. Rev Sci Instr 2001:72:2562-2565, [5] Hudson, LT ef al., Rev Sei Instr (to be published).

[6] Yaakobi, B, Marshall, FJ, Epstein, R. High temperature of laser-compressed shells measured with $\mathrm{Kr}^{34+}$ and $\mathrm{Kr}^{35+} \mathrm{X}$-ray lines. Phys Rev $\mathrm{E} 1996 ; 54: 5848-5850$.

[7] Chung, H-K, Morgan, WL, and Lee, RW. FLYCHK: an extension to the K-shell spectroscopy kinetics model FLY. JQSRT 2003;81:107-115. 
Table 1. Calculated Krypton Average Charge.

\begin{tabular}{lll}
\hline & \multicolumn{2}{c}{ Size } \\
& $0 \mathrm{~mm}$ & $1.2 \mathrm{~mm}$ \\
\hline 2.0 & 27.19 & 29.77 \\
2.2 & 27.63 & 30.29 \\
2.4 & 28.15 & 30.80 \\
2.6 & 28.72 & 31.26 \\
2.8 & 29.33 & 31.65 \\
3.0 & 29.93 & 31.98 \\
3.2 & 30.49 & 32.24 \\
3.4 & 30.99 & 32.45 \\
3.6 & 31.41 & 32.61 \\
3.8 & 31.76 & 32.74 \\
\hline
\end{tabular}




\section{Figure Captions}

Fig. 1. Schematic of the HENEX instrument.

Fig. 2. Comparison of gold spectra recorded using a laboratory $\mathrm{x}$-ray source with a gold anode and from a gold half hohlraum irradiated by the LLE Omega laser.

Fig. 3. HENEX spectral images recorded from a $\mathrm{Kr}$-filled gasbag (top) and from a $\mathrm{Kr}$ filled hohlraum (bottom).

Fig. 4. The HENEX spectrum from a $\mathrm{Kr}$-filled gasbag and a $\mathrm{Zn}$ backlighter (BL). The energy positions of the krypton Ka (neutral atom), H-like Ho, and He-like lines are indicated.

Fig. 5. (a) The spectra summed over the unfiltered (saturated) and filtered (unsaturated) portions of the HENEX spectral image. (b) The spectra calculated for a $2 \times 10^{19} \mathrm{em}^{-3} \mathrm{Kr}$ density and electron temperatures of $2.6 \mathrm{keV}$ to $3.4 \mathrm{keV}$ (bottom to top spectra, respectively). (c) The spectra calculated for $3.0 \mathrm{keV}$ temperature and $\mathrm{Kr}$ densities from $2 \times 10^{17} \mathrm{~cm}^{-3}$ to $2 \times 10^{20} \mathrm{~cm}^{-3}$ (top to bottom spectra).

Fig. 6. The spectra generated by time-dependent calculation performed by assuming that the population is initially in the bare nucleus state at $t=0$ and recombines at a fixed $T_{c}=3$ keV. (a) The spectra calculated for times $0.1 \mathrm{~ns}, 0.2 \mathrm{~ns}, 0.3 \mathrm{~ns}, 0.5 \mathrm{~ns}, 0.7 \mathrm{~ns}$, and $0.9 \mathrm{~ns}$ (top to bottom spectra). (b) The spectra calculated at times $1 \mathrm{~ns}, 2 \mathrm{~ns}, 3 \mathrm{~ns}, 4 \mathrm{~ns}$, and $5 \mathrm{~ns}$. The spectra calculated for 3 ns through 5 ns are very similar and are practically indistinguishable.

Fig. 7. The spectra recorded by a Ge(220) transmission crystal from a laboratory x-ray source with a tungsten anode. 
Fig. 8. The design of a spectrometer using a Ge(220) crystal to record the gold K-shell emission. $\mathrm{Re}=$ radius of curvature. 


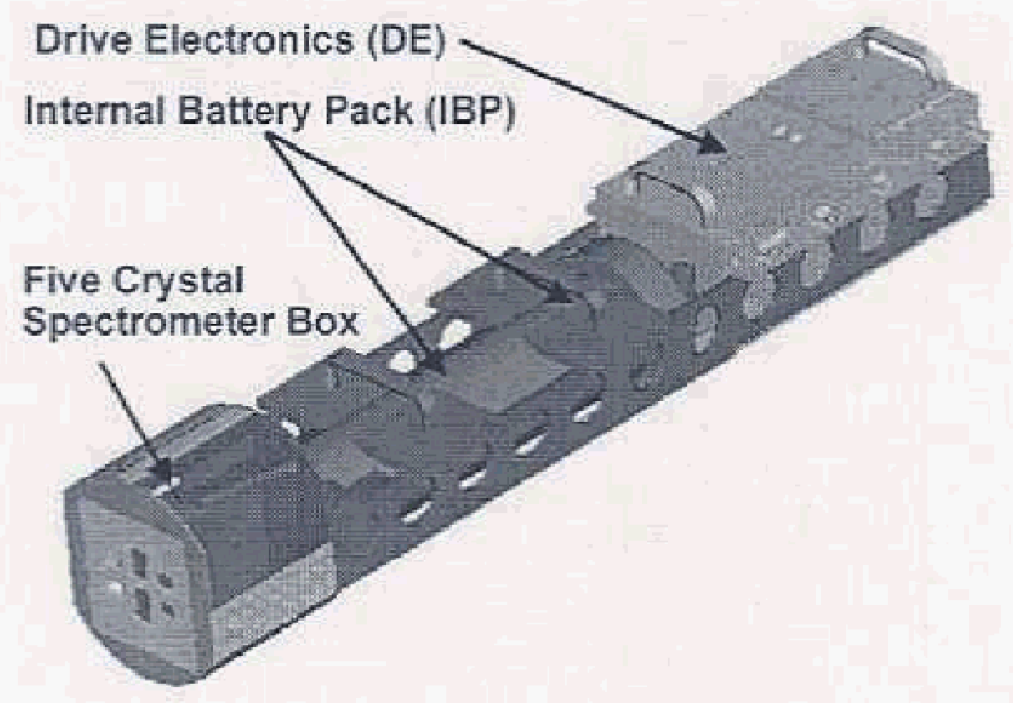

Figure 1 


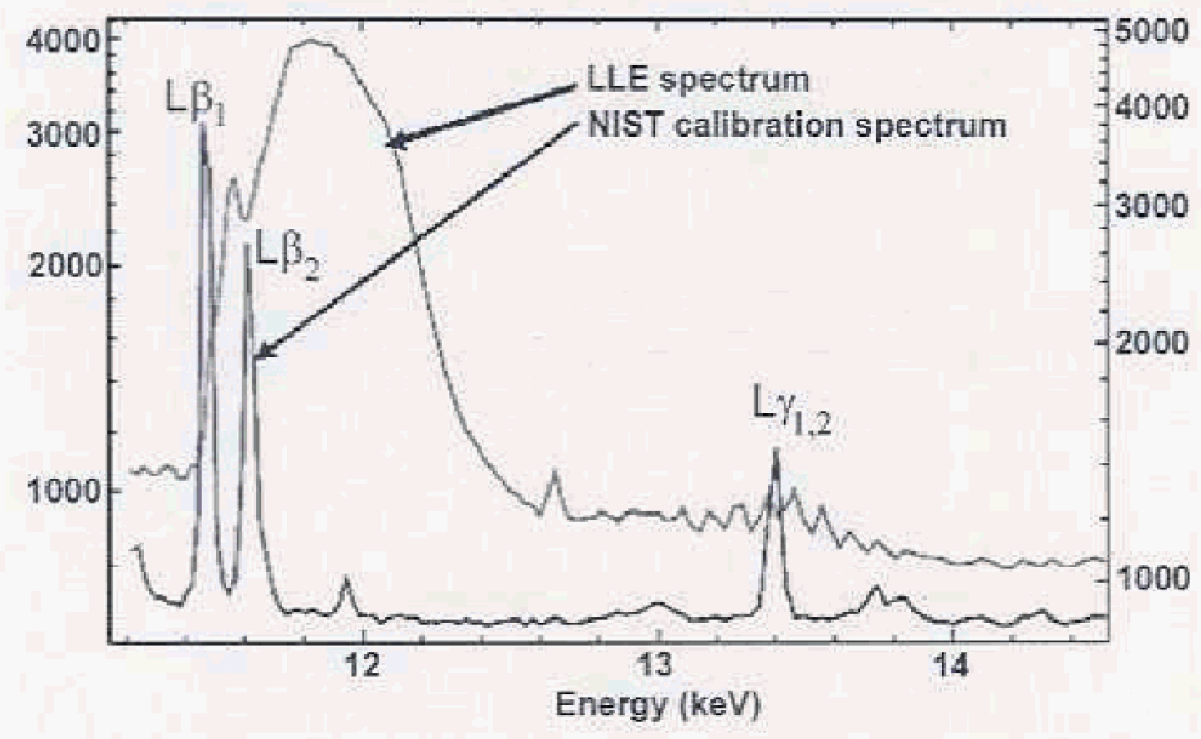

Figure 2 


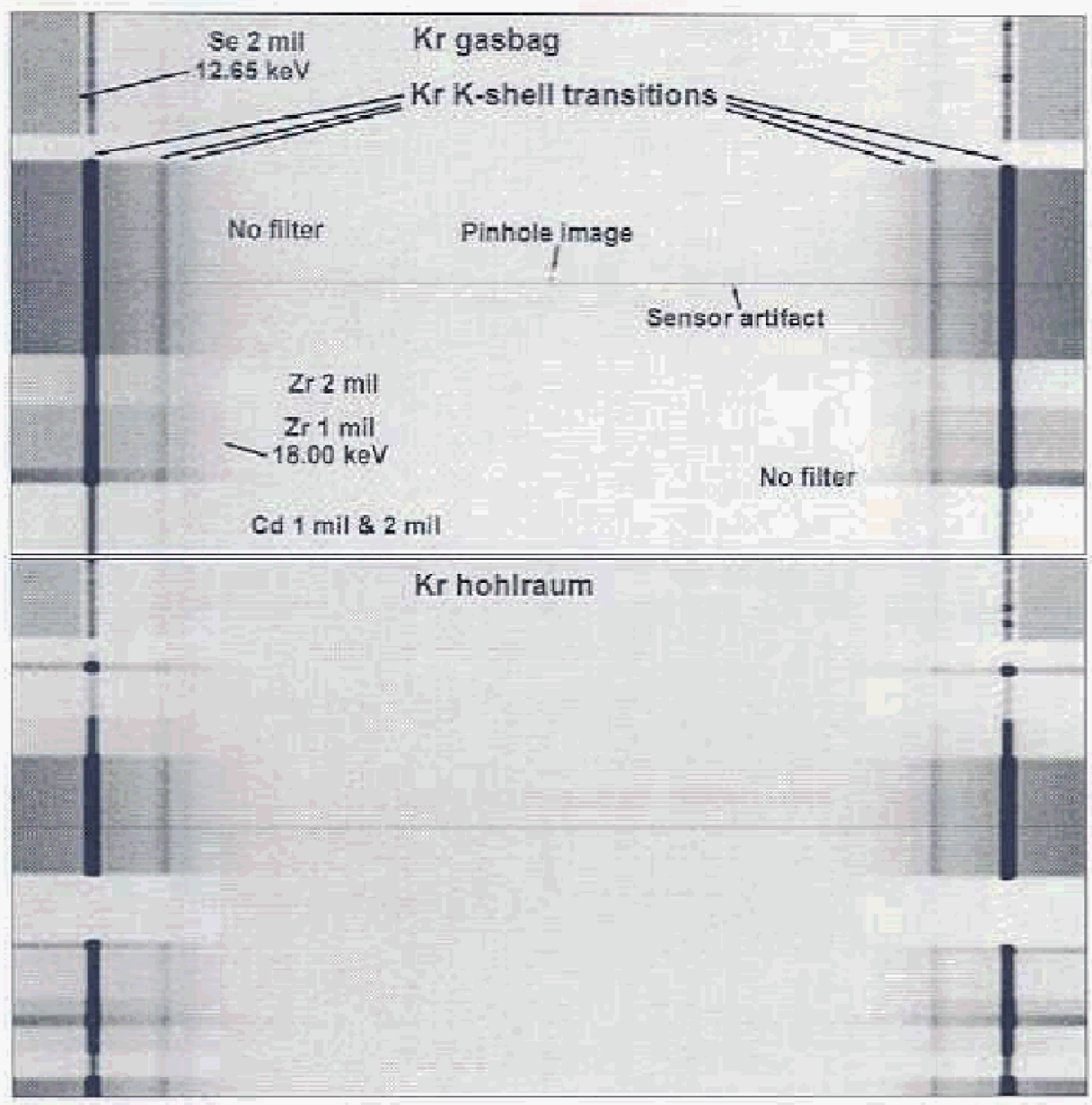

Figure 3 


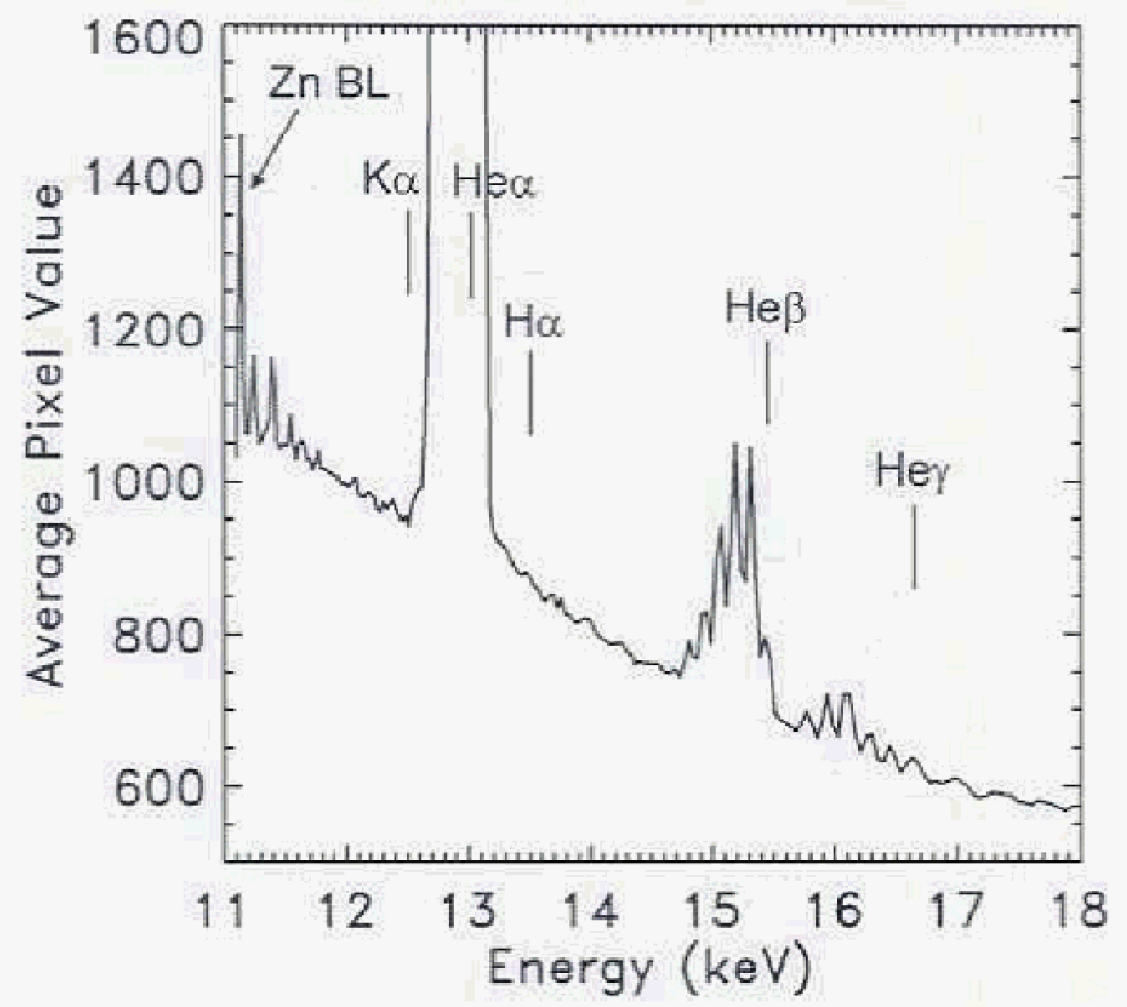

Figure 4 


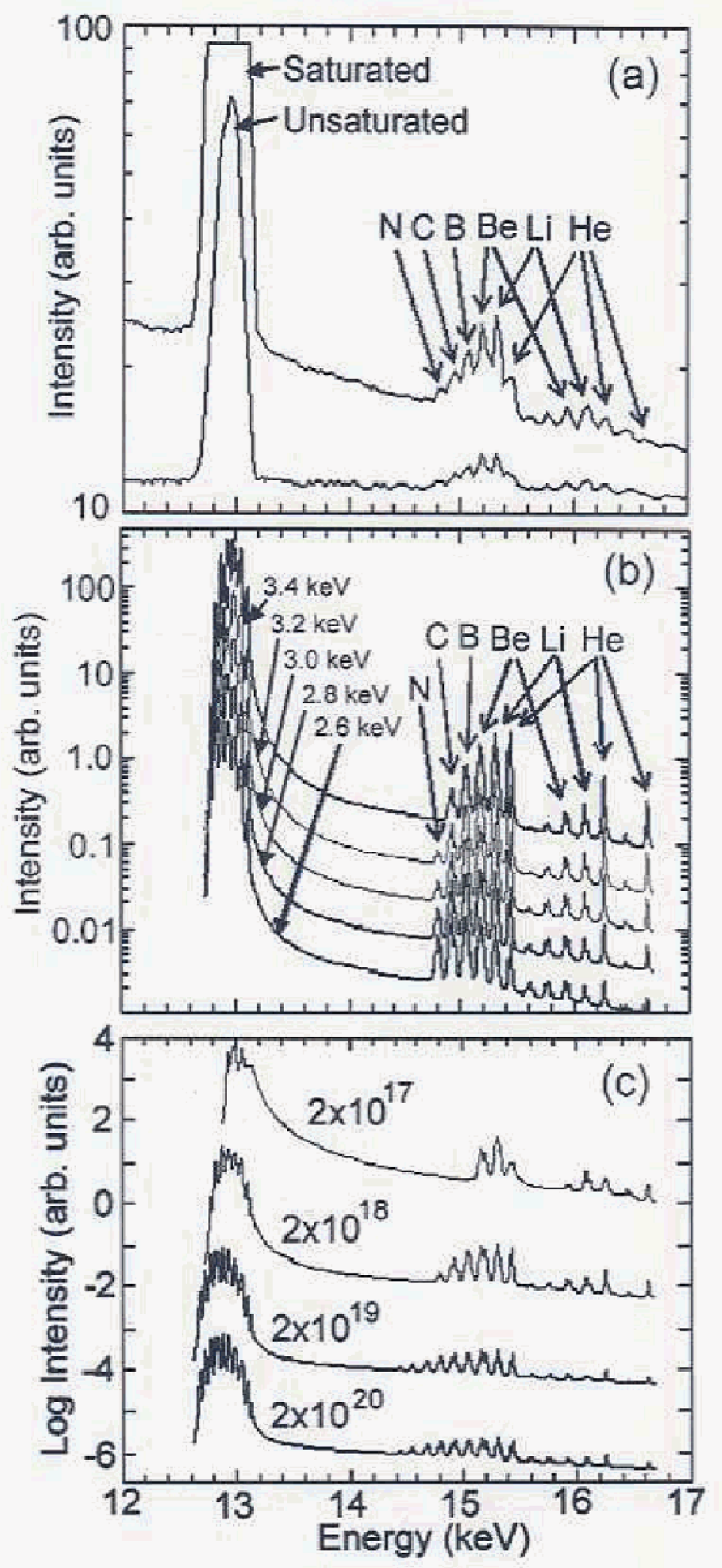

Figure 5 


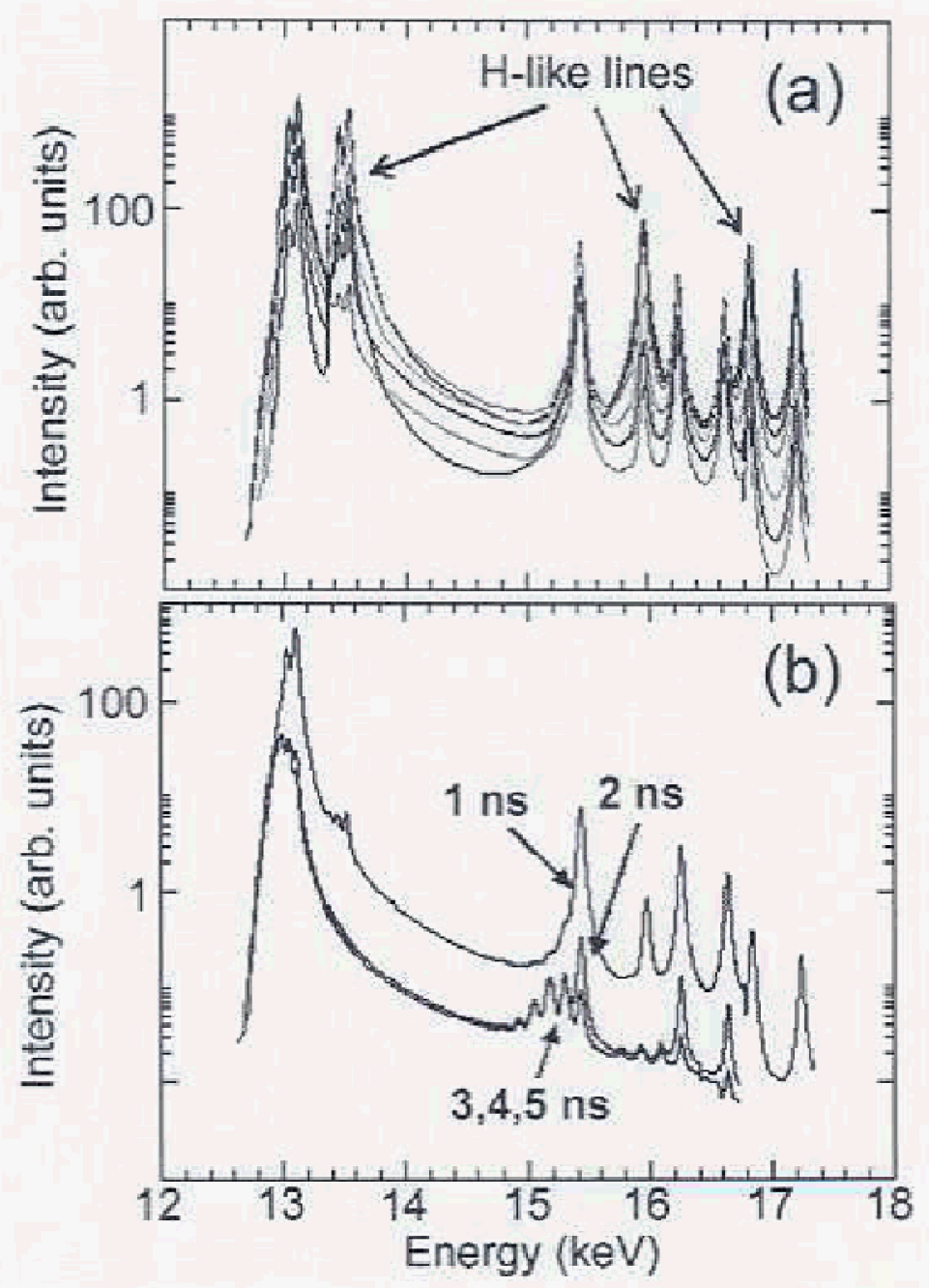

Figure 6 


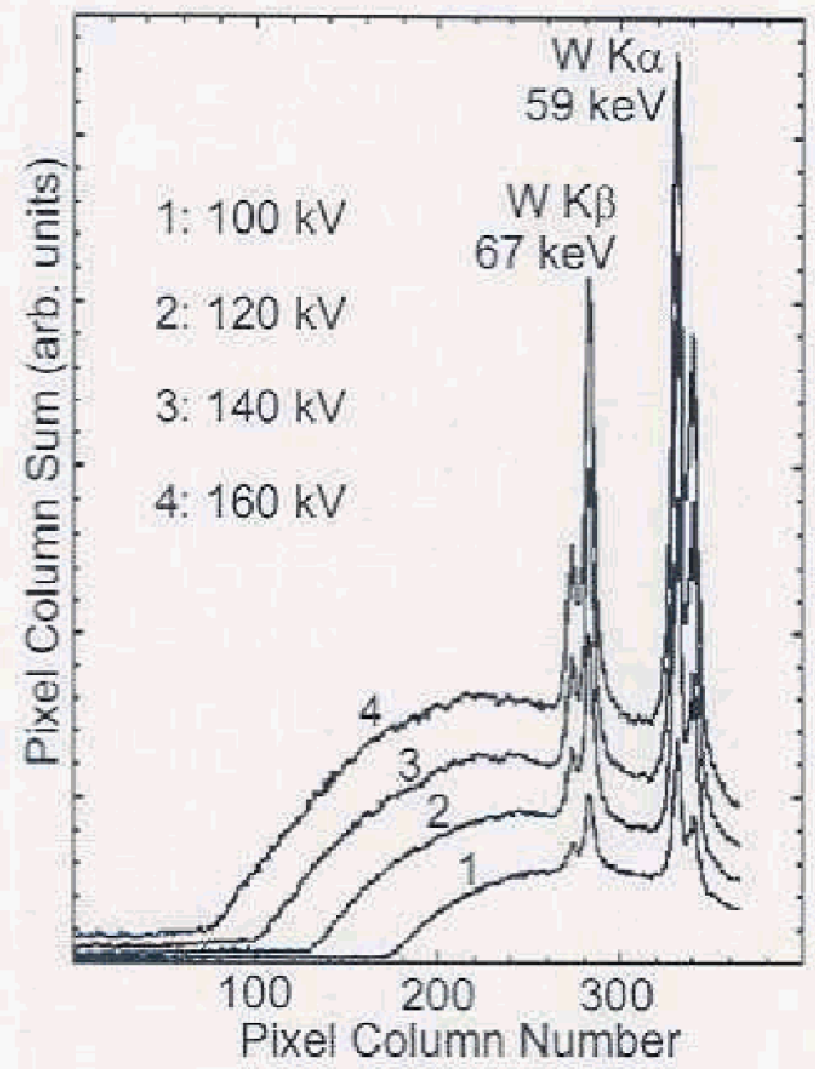

Figure 7 


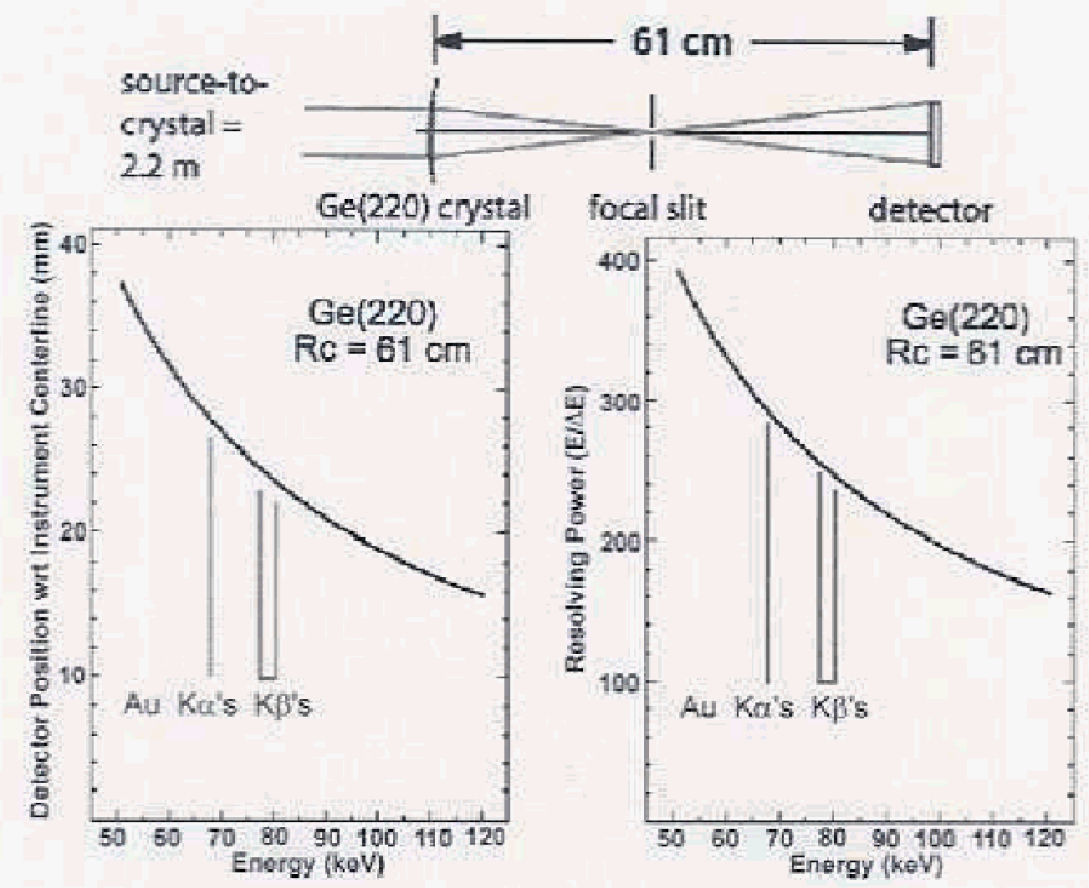

Figure 8 